\title{
THE
}

3-18-2011

\section{Beams of Gravitationally Bound Ultracold Neutrons in Rough Waveguides}

Mauricio Escobar

University of Rhode Island

A. E. Meyerovich

University of Rhode Island, sfo101@uri.edu

Follow this and additional works at: https://digitalcommons.uri.edu/phys_facpubs

Terms of Use

All rights reserved under copyright.

\section{Citation/Publisher Attribution}

Escobar, M., \& Meyerovich, A. E. (2011). Beams of gravitationally bound ultracold neutrons in rough waveguides. Phys. Rev. A, 83, 033618. doi: 10.1103/PhysRevA.83.033618

Available at: http://dx.doi.org/10.1103/PhysRevA.83.033618

This Article is brought to you for free and open access by the Physics at DigitalCommons@URI. It has been accepted for inclusion in Physics Faculty Publications by an authorized administrator of DigitalCommons@URI. For more information, please contact digitalcommons-group@uri.edu. 


\title{
Beams of gravitationally bound ultracold neutrons in rough waveguides
}

\author{
M. Escobar and A. E. Meyerovich \\ Department of Physics, University of Rhode Island, Kingston, Rhode Island 02881-0817, USA
}

(Received 24 January 2011; published 18 March 2011)

\begin{abstract}
We investigate the propagation of ultracold neutrons through a rough waveguide in conjunction with recent experiments in which the ultracold neutrons were beamed between a perfect mirror and a rough scatterer and absorber. The main goal is to find a way to resolve the lowest gravitationally quantized discrete states in the peV range. We compare the neutron count for various types of mirrors with Gaussian, power-law, and exponential correlation functions of surface inhomogeneities. The main conclusion is that all the information about inhomogeneities, including their amplitude, correlation radius, and the rate of decay of the correlation function, enter the exit neutron count via just a single constant $\Phi$, which effectively renormalizes the amplitude of roughness. To observe well-defined quantum steps, one should have an experimental setup with $\Phi>40$. For a wide variety of correlation functions, the constant $\Phi$ is proportional to the square of the amplitude of the surface roughness and is inversely proportional to the square root of the correlation radius. The strong dependence of $\Phi$ on roughness parameters and the shape of the correlation function opens a novel way for improving the resolution of gravitationally bound states by optimizing the roughness pattern without reverting to an undesirable strong roughness. We discuss how to optimize the scatterer and absorber by first generating numerically the desired roughness profile and then transferring it to the mirror. We also study the effect of beam preparation on the initial occupancies of gravitational states before the beam enters the waveguide. It turns out that there are simple ways to manipulate the beam in front of the waveguide that can help to resolve the gravitationally bound quantum states. Our results are in good agreement with available experimental data.
\end{abstract}

DOI: 10.1103/PhysRevA.83.033618

PACS number(s): 03.75.Be, 03.65.Ta, 81.07.St

\section{INTRODUCTION}

The recent observation of the quantization of motion of ultracold neutrons by a gravitational field [1] was one of the most interesting experimental achievements in neutron physics. This is a significant breakthrough in a field with a relatively long theoretical and experimental history going back at least into the late 1960s; for a recent review and a list of publications in the field, see Ref. [2]. The discrete quantum states for neutrons in Earth's gravitational field have extremely low energies with the scale of $1 \mathrm{peV}$. Though the quantization of motion by a linear field such as gravity is not new in and of itself [3] and has already been encountered experimentally in a low-temperature context [4], the experimental access to a spectrum of discrete energy states in such a low-energy range paves the way for using ultracold neutrons as a very sensitive probe for extremely weak fundamental forces [2,5-7].

Currently, the experimental resolution of gravitational states is achieved by sending a horizontal beam of the gravitationally quantized ultracold neutrons between two horizontal mirrors. The top mirror, the "ceiling," is intentionally made rough, while the bottom one, the "floor," is nearly ideal (the quality of this mirror is such that it can ensure thousands of almost specular consecutive reflections [8]). The specularity of reflection is ensured only when the vertical velocity of neutrons is below a certain threshold. The neutrons with the vertical velocities above this threshold are absorbed by the mirrors' material. The beam of ultracold neutrons entering this waveguide consists of neutrons with a relatively large horizontal component of velocity and a much smaller residual vertical component. The quantization of the vertical motion of neutrons by Earth's gravity field corresponds to the quantization of the amplitude of bounces of neutrons from the floor mirror. The scattering of neutrons by the rough ceiling causes turning of the velocity vector and, therefore, a noticeable increase in its vertical component and, eventually, absorption of the scattered neutrons. In quantum language, the turning of the velocity corresponds to the scattering-driven transitions of neutrons into higher quantum states. Only the neutrons in the lowest gravitational states with the lowest kinetic energy of vertical motion and, therefore, the lowest amplitudes of bounces, which could not reach the rough ceiling mirror, continue bouncing unimpeded along the floor mirror and are counted by an exit neutron counter.

Ideally, the exit neutron count for quantized neutrons in such an experiment should have been a step function of a width of the waveguide (the distance between the floor and the ceiling). When the spacing between the mirror decreases, at certain points the spacing becomes equal to the quantized amplitude of neutron bounces, and the neutrons from the corresponding quantum state start experience robust scattering and, eventually, get absorbed. In reality, because of quantum tunneling, the neutrons get scattered before the spacing becomes equal to their amplitude of bounces and the experimental curves are relatively smooth, although the overall quantum cutoff is indisputably clear. The purpose of this paper is twofold. First, we would like to find out what information can be extracted from the existing experimental data that do not exhibit obvious quantum steps. The second question is whether it is possible to improve the resolution of such experiments without considerable changes in the experimental setup. We will look primarily at the possibility of optimizing the roughness of the ceiling mirror and at beam preparation.

Recently [9,10], we developed a theoretical framework for describing experiments with collimated beams of ultracold neutrons in rough waveguides based on our theory of particle 
diffusion along random rough surfaces (see, e.g., a short review in Ref. [11] and references therein) in which the experimental results are linked explicitly to the parameters of the surface roughness. Note that there is also an alternative theoretical approach to the same experiment [12], which, however, does not reveal such an explicit link. The agreement between our theory and experiment was actually better than one would expect [10] given the existing uncertainty in the input parameters. If one is serious about using gravitational quantization of neutrons for precision measurements, the quality of the input parameters should be improved. Below, we will analyze which of the parameters are important for the accurate interpretation of experiments and which ones could be safely ignored. We will also discuss realistic ways for improving experimental data and mitigation of experimental uncertainties.

There are two major uncertainties in experiment. First, the correlation function of surface roughness, which determines neutron scattering by the rough mirror, is unknown. Neither the type of correlation function nor its parameters are known. The only information available from looking at the mirror under the microscope is the approximate value of the average amplitude and lateral size of roughness (the latter parameter can be used for approximating the correlation radius of surface inhomogeneities, assuming that the correlation function is exponential or Gaussian). Since we know that diffusion along rough walls depends on the shape of the correlation function [13], the available information is insufficient for accurate interpretation of the experimental data. In this paper, we will investigate the effect of different types of roughness on the neutron count. We will demonstrate that a better understanding of this issue could open a novel way for improving the resolution of quantized levels: since the mirror roughness is handmade, a proper control and optimization of the roughness pattern can vastly improve the resolution.

The second uncertainty concerns the occupancy of the gravitational states before the beam enters the waveguide. We will demonstrate that the natural assumption that these very low energy levels are uniformly populated can be easily violated depending on the beam preparation. This can not only present a challenge for extracting the data from experiment, but, under certain conditions, it can provide an opportunity for preparing a more desirable initial distribution.

The paper has the following structure. In the next section, we introduce proper dimensionless variables and present the main equations. In Sec. III, we analyze the effect of different types of surface roughness on the exit neutron count for the collimated beam. In Sec. IV, we discuss the effect of the beam preparation on the distribution of neutrons over the gravitational states before the beam enters the collimator. Section $\mathrm{V}$ contains a summary and discussion, including suggestions for future experiments.

\section{QUANTUM DIFFUSION OF NEUTRONS IN A ROUGH WAVEGUIDE}

In this section, we briefly introduce proper dimensionless variables, which are common to the field, and give the main equations. A more detailed introduction can be found in Ref. [10]. In a typical experiment, a beam of ultracold neutrons with energy $E$ goes through a collimator consisting of a perfect mirror on the bottom (floor) and a rough mirror on the top (ceiling). The absorption threshold of the mirror material for neutrons hitting the wall normally is $U_{c}$. The width of the collimator (neutron waveguide) $H$ can be easily changed in a relatively wide range. The main experimental result is the number of neutrons exiting the collimator as a function of $H, E, U_{c}$, and the mirror roughness, although the adjustment of the mirror parameters, with the exception of $H$, means disruptions in experiment.

The distances $z$ are often measured in units of $l_{0}, s=z / l_{0}$, where $l_{0}=\hbar^{2 / 3}\left(2 m^{2} g\right)^{-1 / 3} \sim 5.871 \mu \mathrm{m}$ is the size of the lowest quantum state in the infinite gravitational trap (open geometry without the ceiling). The energies of the quantum levels $\epsilon_{n}$ are measured in units of $e_{0}, \lambda_{n}=\epsilon_{n} / e_{0}$, where $e_{0}=$ $m g l_{0} \sim 0.602 \mathrm{peV} \sim 9.6366 \times 10^{-32} \mathrm{~J}$ is the gravitational energy of a neutron in the same lowest quantum state. In these units, the typical overall kinetic energy of particles in the beam $E$ and the absorption threshold $U_{c}$ are very large, $1.37 \times 10^{5}<e=E / e_{0}<8.65 \times 10^{5}, u_{c}=U_{c} / e_{0} \sim$ $1.4 \times 10^{5}$. This shows that the exact details of the potential well near the absorption threshold are irrelevant as far as the lowest gravitational states $\epsilon_{n}$ are concerned. Changing of energy $e$ in this range corresponds to the change of ratio $\chi=U_{c} / E \equiv u_{c} / e$ in the range

$$
0.16 \lesssim \chi \equiv u_{c} / e<1 .
$$

Parameter $\chi$ shows how easy it is for a neutron to get absorbed by a wall when the direction of velocity is rotated as a result of scattering by the rough mirror. The dimensionless distance between the walls is $h=H / l_{0}$. A more detailed description of the energy spectrum of the gravitationally bound states of neutrons in the collimated beam, including the dependence $\epsilon_{n}(h)$, is given in Ref. [10].

It is also convenient to introduce the dimensionless velocities (momenta) in the beam direction along the wall ( $x$ direction) $\beta_{j}, v_{j}=\beta_{j} v_{0}, v_{0}=\sqrt{2 g l_{0}}=\hbar / m l_{0} \sim 1.073 \times$ $10^{-2} \mathrm{~m} / \mathrm{s}, \beta_{j}=\sqrt{e-\lambda_{j}} \equiv p_{j} l_{0}$ [for lower levels, $\lambda_{j} \ll e$ and $\left.\beta_{j} \simeq \sqrt{e}\left(1-\frac{1}{2} \lambda_{j} / e\right)\right]$.

For the time units, one can use parameter $\tau_{0}$,

$$
\frac{1}{\tau_{0}}=\frac{\sqrt{2 \pi}}{4 m} \frac{\hbar}{l_{0}^{2}} \approx 1148.7 \mathrm{~s}^{-1},
$$

which provides the scale for the oscillation frequency of neutrons in the gravitational well. In experiment [1], the typical time of flight of neutrons through the cell is $t=L_{x} / V_{0} \sim$ $2 \times 10^{-2} \kappa \mathrm{s}$ (parameter $\kappa$ is close to, but not always equal to, 1 because of variations in the beam energy $u_{c} / \chi$ and in the cell length). Then

$$
t / \tau_{0} \simeq 23 \kappa
$$

In numerical examples we use $\kappa=1$, although this parameter could be easily adjusted (in experiment, of course, the length of the collimator is fixed).

Since one of our main goals here is to explore the effect of different types of mirror roughness on the exit count for neutrons in various gravitational states, we will try to work 
as long as possible with the correlation function of surface inhomogeneities of a general form,

$$
\zeta(x)=\ell^{2} \varphi(x / R),
$$

and specify a particular form of the function $\varphi$ later. The average amplitude and the correlation radius of surface roughness $\ell$ and $R$ in our new variables are $\eta=\ell / l_{0}$ and $r=R / l_{0}$. More important than the correlation function itself is its Fourier image, the so-called power spectrum of surface roughness, which in our dimensionless variables assumes the form

$$
\begin{gathered}
\zeta\left(p_{j}-p_{j^{\prime}}\right)=\sqrt{2 \pi} l_{0}^{3} \gamma^{2} r^{3} \psi(y), \\
y=\left(\beta_{j}-\beta_{j^{\prime}}\right) r,
\end{gathered}
$$

where $\gamma$ is the aperture of roughness,

$$
\gamma=\ell / R \equiv \eta / r .
$$

The roughness is weak when both its amplitude and its aperture are small,

$$
\eta \ll r, h .
$$

The most widely used form of the correlation function in theoretical calculations is the Gaussian function, which has the Gaussian power spectrum as well:

$$
\begin{gathered}
\varphi_{G}(x / R)=\exp \left(-x^{2} / 2 R^{2}\right), \\
\psi_{G}(y)=\exp \left(-y^{2} / 2\right) .
\end{gathered}
$$

This is the correlator that we used in our earlier work on neutrons $[9,10]$. There are many other realistic forms of the correlation function that differ from each other by the rate of decay of the correlator in real or Fourier space [14-16]. Below, in addition to the Gaussian correlator, we will investigate two major classes of the correlation functions following Ref. [13]. These include the correlators with various power-law decays in real space,

$$
\begin{aligned}
\varphi_{\mu}(x / R) & =2 \mu /\left(1+x^{2} / R^{2}\right)^{1+\mu}, \\
\psi_{\mu}(y) & =\frac{y^{\mu}}{2^{\mu-1} \Gamma(\mu)} K_{\mu}(y),
\end{aligned}
$$

with a wide range of values for the index $\mu$, and the correlators with the power-law decay in Fourier space,

$$
\begin{gathered}
\psi_{\lambda}(y)=1 /\left(1+y^{2}\right)^{1+\lambda}, \\
\varphi_{\lambda}(x / R)=\frac{(x / R)^{\lambda}}{2^{\lambda} \Gamma(1+\lambda)} K_{\lambda}(x / R)
\end{gathered}
$$

with various values of $\lambda$. Note that the Bessel functions $K_{\mu}$ and $K_{\lambda}$ are in essence exponentially attenuating functions; these functions acquire a pure exponential form at $\mu=\lambda=1 / 2$,

$$
K_{1 / 2}(x)=\sqrt{\frac{\pi}{2}} \frac{e^{-x}}{\sqrt{x}} .
$$

Note that the attribution of a particular form of the roughness correlation function to a rough surface goes beyond just choosing functions $\varphi, \psi$. For example, a fit of the scanning tunneling microscopy (STM) data by the Gaussian (9) and exponential (14) correlators $(\lambda=1 / 2)$ in Ref. [16] showed that both could account for the measured roughness profile. However, the sets of values of the average amplitude of roughness $\ell$ and the correlation radius $R$, which were extracted from the fit of the experimental profile to these two correlators, were significantly different. The most likely reason is an insufficiently large size of the surface sample in typical STM measurements. This means that one should be very cautious when ascribing the experimental values of the average height and lateral size of inhomogeneities to some ad hoc correlation function as its amplitude and correlation radius $\ell$ and $R$.

In Ref. [10], we demonstrated that scattering by a rough mirror leads to diffusion of neutrons over the discrete gravitational states and that this diffusion has a strong directional bias upward, toward higher and higher states. The roughnessdriven transition probabilities between the states $j, j^{\prime}$ are $[9,11]$

$$
\frac{1}{\tau_{j j^{\prime}}}=v_{j}^{-1} \zeta\left(p_{j}-p_{j^{\prime}}^{\prime}\right) U_{c}^{2} \Psi_{j}^{2}(H) \Psi_{j^{\prime}}^{2}(H),
$$

where $\Psi_{j}(H)$ is the value of the wave function of neutrons in the gravitational state $j$ on the rough wall. The bias is explained by the rapid growth of $\Psi_{j}(H)$ with increasing $j$ and $j^{\prime}$ (roughly, as $j^{2} j^{\prime 2}$ ). This increase in the rate of jumps $j \rightarrow j^{\prime}$ with increasing $j^{\prime}$ is cut off by the attenuation of the correlation function $\zeta\left(p_{j}-p_{j^{\prime}}^{\prime}\right)$ at large $\left|p_{j}-p_{j^{\prime}}^{\prime}\right|$, which, in turn, is determined by the value of the correlation radius $R$. As a result, the transition rates $1 / \tau_{j j^{\prime}}$ for transitions $j \rightarrow j^{\prime}$ represent, as a function of $j^{\prime}$, a relatively narrow peak centered around some value $j_{1} \gg j$. This bias in transition rates is so strong that almost all time $\tau_{j}$ necessary for a neutron, which is initially in some low gravitational state $j$, to go up in states and, eventually, to go over the absorption barrier $U_{c}$, is spent on the first transition upward.

Since the center of the peak for transitions from $j$ to $j^{\prime}$ is located at some $j_{1} \gg j$ and the peak is very high, the absorption times $\tau_{j}$ for neutrons that initially occupy some of the lowest gravitational states $j$ differ from each other, according to Eq. (16), only by the values $\Psi_{j}^{2}(H)$. In the end [10],

$$
\frac{1}{\tau_{j}}=\frac{b_{j}}{b_{1}} \frac{1}{\tau_{1}},
$$

where $\tau_{1}$ is the absorption time for neutrons in the firstthe lowest-gravitational state, and $b_{j}$ are the dimensionless values of $\Psi_{j}^{2}(H)$,

$$
b_{j} \equiv 10^{5} l_{0} \Psi_{j}^{2}(H) / 2
$$

(the coefficient $10^{5}$ is inserted purely for computational convenience). The absorption rate $1 / \tau_{1}$, scaled over the typical rate $1 / \tau_{0}$, Eq. (2), is a sum of two parts: the scattering rate $w_{1}^{(0)}$ directly into continuum above the threshold $U_{c}$ and the rate of absorption via diffusion over discrete states $w_{1}^{(1)}$. Since such diffusion has a very strong upward bias and accelerates with each jump, $w_{1}^{(1)}$ is simply a sum of the transition rates from the state $j=1$ into any of the upper states:

$$
\frac{1}{\tau_{1}}=\frac{1}{\tau_{0}}\left(w_{1}^{(0)}+\sum_{i>1} w_{1 i}\right) .
$$


In our notations,

$$
w_{1}^{(0)}=2 \times 10^{-5} \gamma^{2} u_{c}^{2} b_{1}(h) F_{0}(\chi, r), \quad \chi=u_{c} / e, \quad \gamma=\eta / r
$$

$$
\begin{aligned}
& \sum_{i>1} w_{1 i}=2 \times 10^{-5} \gamma^{2} u_{c}^{2} b_{1}(h) F_{1}(r, h), \\
& F_{0}(\chi, r)=\frac{2 r^{3} \sqrt{\chi}}{\pi} \int_{0}^{1 / \chi-1} \frac{d z}{\sqrt{z+1}} \frac{\psi(y)}{3+1 / z}, \\
& y=(\sqrt{1 / \chi}-\sqrt{1 / \chi-1-z}) \sqrt{u_{c}} r, \\
& F_{1}(r, h)=2 \times 10^{-5} \frac{r^{3} \sqrt{\chi}}{\sqrt{u_{c}}} \sum_{\lambda_{i}<u_{c} / \chi} b_{i}(h) \psi\left(y_{i}\right) \text {, } \\
& y_{i}=\left(\sqrt{1 / \chi}-\sqrt{1 / \chi-\lambda_{i} / u_{c}}\right) \sqrt{u_{c}} r .
\end{aligned}
$$

Then

$$
\tau_{0} / \tau_{1}=2 \times 10^{-5} b_{1} u_{c}^{2} \gamma^{2}\left[F_{0}(\chi, r)+F_{1}(r, h)\right],
$$

where the first term describes the direct transitions over the absorption threshold, and the second describes the strongly biased upward diffusion. The neutron count on the exit counter is equal to

$$
\begin{aligned}
N_{e} & =\sum N_{j}(0) \exp \left(-L / v_{j} \tau_{j}\right) \\
& =\sum N_{j}(0) \exp \left(-L b_{j} / b_{1} \sqrt{e} v_{0} \tau_{1}\right) \\
& =\sum N_{j}(0) \exp \left(-23 \kappa \tau_{0} b_{j} / b_{1} \tau_{1}\right) \\
& \simeq \sum N_{j}(0) \exp \left(-46 \times 10^{-5} \gamma^{2} \kappa u_{c}^{2} F_{1} b_{j}\right),
\end{aligned}
$$

where $N_{j}(0)$ is the initial occupancy of the level $j$. Although in experiments [1] parameter $\kappa=1$, we keep this parameter as long as we can since the length of the cell $L$ or the speed of the beam $v_{0}$ can change in future experiments. If all initial level occupancies are the same, $N_{j}(0)=N_{0}$, then

$$
\begin{gathered}
N_{e}=N_{0} f(r, h), \\
f(r, h)=\sum \exp \left(-46 \times 10^{-5} \gamma^{2} \kappa u_{c}^{2} F_{1} b_{j}\right) .
\end{gathered}
$$

If, as is indicated by some of the experiments [1], the initial population of the first level is about one-half of the occupancy of the higher ones, the function $f$ should be replaced by

$$
\begin{aligned}
f_{2}(x)= & \frac{1}{2} \exp \left(-46 \times 10^{-5} \gamma^{2} \kappa u_{c}^{2} F_{1}\right) \\
& +\sum_{j>1} \exp \left(-46 \times 10^{-5} \gamma^{2} \kappa u_{c}^{2} F_{1} b_{j}\right) .
\end{aligned}
$$

We think that the main unresolved issue facing the experiment is the lack of accurate information about the occupancy of the low gravitational levels before the beam enters the rough waveguide.

\section{NEUTRON COUNT FOR DIFFERENT TYPES OF ROUGHNESS}

\section{A. Simple analytical results}

Under normal experimental conditions, the correlation radius of the mirror's roughness $r$ is not very large. Then the peak in the dependence of the roughness-driven jump rates $1 / \tau_{j j^{\prime}}$ on $j^{\prime}$ for transitions $j \rightarrow j^{\prime}$, which originate from the lowest gravitational states $j$, is located at large values of $j^{\prime}$, $1 \ll j^{\prime} \ll u_{c}$. This leads to three important conclusions. First, the destination states $j^{\prime} \gg 1$ are far away from the bottom of the well where the presence of the gravitational field is important and the states within the peak can be described within the deep square-well approximation, Ref. [9]:

$$
\begin{gathered}
\lambda_{j^{\prime}}=\pi^{2} j^{2} / h^{2}, \\
\Psi_{j^{\prime}}^{2}(0)=\Psi_{j^{\prime}}^{2}(H)=\frac{2}{l_{0} h} \frac{\lambda_{j^{\prime}}}{u_{c}}, \quad b_{j^{\prime}} \equiv 10^{5} \frac{\lambda_{j^{\prime}}}{h u_{c}} .
\end{gathered}
$$

In this case, $F_{1}(r, h)$, Eq. (23), becomes

$$
F_{1}(r, h)=2 \frac{\pi^{2} r^{3} \sqrt{\chi}}{h^{3} u_{c}^{3 / 2}} \sum_{n>1}^{S} n^{2} \psi\left(y_{n}\right)
$$

with

$$
S=\operatorname{Int}\left[(h / \pi) \sqrt{u_{c} / \chi}\right] \gg 1 .
$$

Second, under the same conditions, the rate of direct over-thethreshold jumps $F_{0}$ is negligible in comparison to the result of the biased upward diffusion $F_{1}$ and can be disregarded, as has already been done in Eqs. (25)-(27).

The third conclusion is that since the only important contributions into the sum (30) come from the terms with $n \gg 1$, the summation can be replaced by the integration,

$$
\begin{aligned}
F_{1}(r, h) & =2 \frac{\pi^{2} r^{3} \sqrt{\chi}}{h^{3} u_{c}^{3 / 2}} \int_{0}^{h \sqrt{u_{c}} / \pi \sqrt{\chi}} n^{2} \psi\left(y_{n}\right) d n \\
& =\frac{2 r^{3}}{\pi \chi} \int_{0}^{1} z^{2} \psi(y) d z, \\
y & =\frac{1}{\sqrt{\chi}}\left(1-\sqrt{1-z^{2}}\right) \sqrt{u_{c}} r .
\end{aligned}
$$

Equation (32) has very serious implications. It means that the width of the neutron waveguide $h$ disappears from the function $F_{1}$, which now depends only on the correlation radius $r$ (and, of course, on the profile of the correlation function $\psi$ ). As a result, $h$ enters the exit neutron count (25)-(27) only via the values of the wave function on the rough mirror $b_{j}(h)$, Eq. (18). To emphasize this point, we can rewrite Eq. (25) for the neutron exit count as

$$
\begin{gathered}
N_{e}=\sum N_{j}(0) \exp \left[-\Phi b_{j}(h)\right], \\
\Phi(\eta, r)=L / \sqrt{e} v_{0} \tau_{1} b_{1}=A \eta^{2} r \int_{0}^{1} z^{2} \psi(y) d z,
\end{gathered}
$$

where the constant $A$ is equal to

$$
A=92 \times 10^{-5} \kappa u_{c}^{2} / \pi \chi
$$

and $y$ is given by Eq. (32). The product $\eta^{2} r$ is not included into the constant $A$ in order to separate parameters of roughnessits amplitude $\eta$, correlation radius $r$, and the function $\psi-$ from other properties of the experimental setup such as the waveguide length $L$, the beam velocity $v_{0}$, the penetration barrier for the neutrons $u_{c}$, and the ratio of the kinetic energy to this barrier $\chi$, Eq. (1). As a reminder, in experiments [1] $\kappa=1, \chi=0.16, u_{c}=1.4 \times 10^{5}$, and, therefore, the value of 


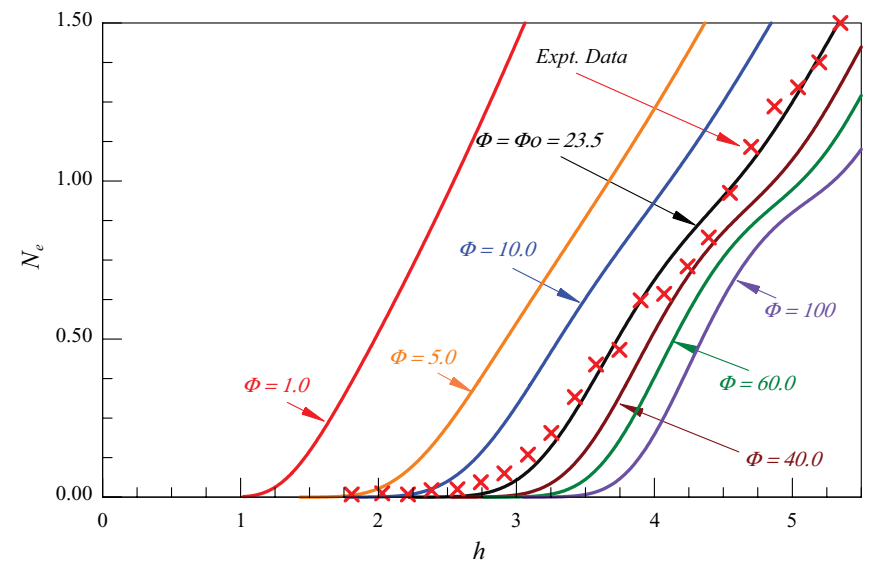

FIG. 1. (Color online) Exit neutron count, Eq. (33), as a function of the distance between the mirrors for different values of $\Phi$ for initial occupancy of quantized states in front of the waveguide chosen as $N_{j}(0)=N_{0}=1$. The curves are marked by the values of $\Phi$ used in the calculations. The curve with $\Phi_{0}=23.48$ is equivalent to the theoretical fit to experimental data of Ref. [1] that was performed in Ref. [10] on the basis of exact equations with the Gaussian correlator and experimental values of parameters $r=1.19, \eta=0.119, \kappa=1$, and $\chi=0.16$. The fitted experimental data are also presented in the figure as crosses. The fit uses $N_{0}$ as a fitting parameter and takes into account the uncertainty of about $20 \%$ in the experimental values of $h$.

$A$ was $A=3.53 \times 10^{7}$. Although this value of $A$ appears to be large, in the end the physically important constant, $\Phi$, which corresponds to experimental data, acquires a "normal" order of magnitude, somewhere between 1 and 100 .

This result shows that the exit neutron count is a universal function of roughness (see also comments in Sec. V). All the information about the correlation function of mirror roughness, including its average amplitude $\eta$ and the correlation radius $r$, and the information about the experimental setup, including the mirror length $L$, beam velocity, and the absorption threshold $u_{c}, \chi$, collapse into a single dimensionless constant $\Phi$, Eqs. (33) and (34). A set of the curves $N_{e}(h)$ for various values of this constant $\Phi$ is given in Fig. 1 assuming that the initial populations of all quantized levels were the same, $N_{j}(0)=N_{0}=1$. This choice $N_{0}=1$ has an additional benefit of having the plotted values for the exit count $N_{e}$ show directly how many levels contribute at each value of the waveguide width $h$. The change in value of $N_{0}$ from the value $N_{0}=1$ would translate simply into a change in the vertical scale in Fig. 1. It is clear from Fig. 1 that one has to have an experimental setup with $\Phi>40$ in order to see well-developed quantum steps.

The curve with $\Phi_{0}=23.48$ in Fig. 1 is indistinguishable from the theoretical fit to the experimental data in Ref. [10]. This fit in Ref. [10] was done numerically starting from Eq. (19) using the Gaussian correlations with parameters $r=$ $1.19, \eta=0.119, \kappa=1$, and $\chi=0.16$. The fitted experimental data are also shown in Fig. 1 as crosses. Note that these values of $r$ and $\eta$ in Ref. [10] were not the fitting parameters, but were the observed values of the amplitude and lateral size of surface roughness in experiment [1]. The only fitting parameter was the value of $N_{0}$ under the assumption that all $N_{j}(0)=N_{0}$, or, in other words, the choice of the vertical scale for plotting the experimental data in this figure. Unfortunately, there was also a noticeable uncertainty-up to $20 \%$-in the measured width of the waveguide $h$, which allowed adjustments in the position of the experimental data. Therefore, in the absence of the accurate experimental information on the values of $N_{0}$ and $h$, the fit in Fig. 1 cannot yet be considered final: there remains a certain flexibility in fitting the same experimental data to the curves with various values of $\Phi$ in the range from about 10 to 40. However, even the mere possibility of a good fit is a clear sign of consistency between theory and experiment.

The only remaining theoretical task is to calculate $\Phi$ for various correlation functions. Since in experiment the threshold $u_{c}$ for neutron penetration into the mirror is very high, $u_{c} \sim 10^{5}$, the integrand in Eq. (32) at any reasonable values of the correlation radius $r$ is a relatively sharp narrow peak with a maximum around $z_{0}^{2} \sim 1 / r \sqrt{u_{c}} \ll 1$ (the exact value of $z_{0}$ depends, of course, on the correlation radius). Therefore, it is possible to expand $\psi(y)$ in the integrand (32) near $z_{0}$ and perform the integration within the peak area. In the case of the Gaussian correlator, Eq. $(10), \psi_{G}(y)=$ $\exp \left(-y^{2} / 2\right)$

$$
\begin{aligned}
\Phi_{G} & =A \eta^{2} r \int_{0}^{1} z^{2} e^{-y^{2} / 2} d z \\
& \simeq \frac{A \eta^{2}}{r^{1 / 2}} \frac{(8 \chi)^{3 / 4}}{3 u_{c}^{3 / 4}} \Gamma\left(\frac{7}{4}\right) .
\end{aligned}
$$

The curve $\widetilde{\Phi}_{G}(r) \equiv \Phi_{G}(r) / A \eta^{2}$ is plotted in Fig. 2 as a function of $r$.

In the case of power-law correlations in momentum space with different indices $\lambda$, Eq. (14), the same approximation yields

$$
\begin{aligned}
\Phi_{\lambda} & =A \eta^{2} r \int_{0}^{1} \frac{z^{2} d z}{\left(1+y^{2}\right)^{1+\lambda}} \\
& \simeq \frac{1}{3} A \eta^{2} r_{2} F_{1}\left(\frac{3}{4}, \lambda+1, \frac{7}{4},-r^{2} u_{c} / 4 \chi\right) \\
& \simeq \frac{A \eta^{2}}{r^{1 / 2}} \frac{(4 \chi)^{3 / 4}}{3 u_{c}^{3 / 4}} \frac{\Gamma(\lambda+1 / 4)}{\Gamma(\lambda+1)} .
\end{aligned}
$$

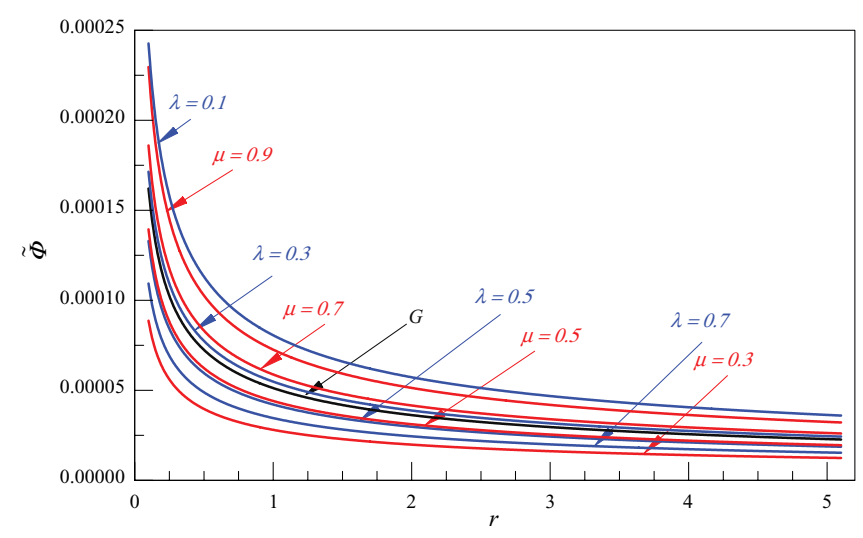

FIG. 2. (Color online) Functions $\widetilde{\Phi}(r)$ for different correlation functions. $\widetilde{\Phi}_{G}$ (black) corresponds to the Gaussian correlator (9), $\widetilde{\Phi}_{\mu}$ (red) to power-law correlators (11), and $\widetilde{\Phi}_{\lambda}$ (blue) to power-law correlators in Fourier space (13). The curves are marked by the values of $\lambda$ and $\mu$. 
The factor $\Gamma(\lambda+1 / 4) / \Gamma(\lambda+1)$ decreases monotonically with increasing $\lambda$ from $\Gamma(1 / 4) \approx 3.63$ at $\lambda=0$ to zero approximately as $1 / \lambda^{3 / 4}-0.094 / \lambda^{7 / 4}$. Functions $\widetilde{\Phi}_{\lambda}(r) \equiv$ $\Phi_{\lambda}(r) / A \eta^{2}$ are plotted in Fig. 2 for several values of $\lambda$. It is important that the Gaussian and power-law power spectra of the correlation function yield the same power of the correlation radius $r$ in the function $\Phi(r)$.

For power-law correlators in real space, Eq. (12), the integration is somewhat more cumbersome because of the presence of the Bessel functions in the integrand. For example, for $\mu=1 / 2$,

$$
\Phi_{\mu=1 / 2}=\frac{A \eta^{2}}{r^{1 / 2}} \frac{\chi^{3 / 4}(\pi / 2)^{1 / 2}}{u_{c}^{3 / 4}}
$$

and $\Phi_{\mu=1 / 2}(r \rightarrow \infty)$ goes to zero as $1 / r^{1 / 2}$, the same as $\Phi_{\lambda}(r \rightarrow \infty)$. We can also give an analytic expression for the asymptotic behavior of $\Phi_{\mu}(r)$ at large $r$ for an arbitrary $\mu$. At large values of the argument, the first term in the asymptotic expansion for all the modified Bessel functions $K_{\mu}(x \gg 1)$ is the same,

$$
K_{\mu}(x \gg 1) \simeq K_{\mu=1 / 2}(x \gg 1),
$$

and

$$
\Phi_{\mu}(r \gg 1)=\frac{A \eta^{2}}{r^{1 / 2}} \frac{2^{1-\mu} \chi^{3 / 4}}{(\mu+1) \Gamma(\mu) u_{c}^{3 / 4}} .
$$

The plots of $\widetilde{\Phi}_{\mu}(r) \equiv \Phi_{\mu}(r) / A \eta^{2}$ for various values of $\mu$ are also shown in Fig. 2.

As is clear from Fig. 2, functions $\widetilde{\Phi}_{\lambda}(r)$ and $\widetilde{\Phi}_{\mu}(r)$ depend differently on their parameters $\lambda$ and $\mu$. This difference in dependencies can result in a change in the hierarchy of values between $\widetilde{\Phi}_{G}(r), \widetilde{\Phi}_{\lambda}(r)$, and $\widetilde{\Phi}_{\mu}(r)$ calculated using the same values of $\eta$ and $r$. To emphasize this point, in Fig. 3 we present $\widetilde{\Phi}_{\lambda}(r=1.19)$ and $\widetilde{\Phi}_{\mu}(r=1.19)$ as functions of $\lambda$ and $\mu$, respectively. The horizontal line corresponds to the value $\Phi_{G}(r=1.19) / A \eta^{2}$. Since we are interested in having a setup with as large a value of the constant $\Phi$ as possible, the fact that different correlation functions dominate in different parameter domains can have important experimental implications.

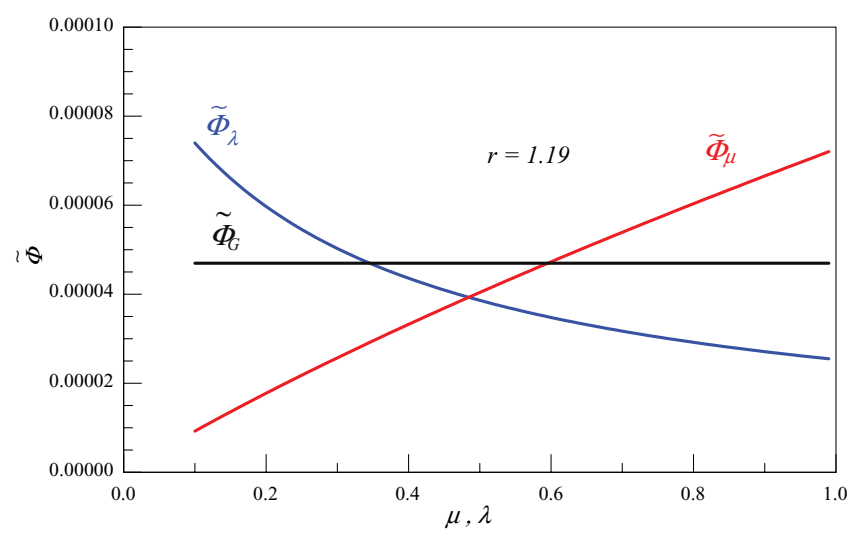

FIG. 3. (Color online) Dependence of the functions $\widetilde{\Phi}_{\lambda}$ (blue curve) on $\lambda$ and $\widetilde{\Phi}_{\mu}$ (red curve) on $\mu$ at $r=1.19$. For comparison, the black horizontal line presents the value of $\widetilde{\Phi}_{G}$ for the same value of $r=1.19$.

\section{BEAM PREPARATION}

Our equations for the exit neutron count (26) assume that the populations of low gravitational states $N_{j}(0)$ are the same before the beam enters the rough waveguide (space between the mirrors). The distribution function of neutrons coming out of the reactor should indeed be flat in the peV energy range. However, before entering the waveguide, the beam goes through a complicated experimental setup, which includes all sorts of splitters, collimators, and other mechanical obstacles. The presence of these obstacles can distort the initially uniform distribution of neutrons over gravitational states.

The simplest example of such distribution distortion is illustrated in Fig. 4. In this figure, we analyze the occupancy of the three lowest gravitational states after the neutron beam, in which occupancy of all states is equal to 1 , encounters an up or down step on the "floor" as a function of the height of the step in units of $l_{0}=\hbar^{2 / 3}\left(2 m^{2} g\right)^{-1 / 3} \sim 5.871 \mu \mathrm{m}$. The calculation was done using the quasiclassical quantization of the gravitational states in open space (no "ceiling"). As is clear from Fig. 4, the populations of the states remain more or less the same if the floor shifts up. However, the level occupancies change dramatically if the beam encounters the step down.

The transition of a neutron to a higher state after a neutron passes over a step down was analyzed in Ref. [17]. The same effect causes the depletion of the population of the lower states in our Fig. 4 for the beam passing over the step down. It is easy to understand qualitatively, even from purely classical considerations, why the number of neutrons with low amplitudes of bounces in the gravitational field decreases when the neutrons pass over the step down-the amplitudes of bounces from a new, lower floor should, of course, exceed those for the initial floor. We do not yet have a simple qualitative explanation for the most intriguing feature in Fig. 4-the presence of distinct quantum plateaus on the occupancy curves. The presence of these plateaus can help to stabilize, if desirable, the occupancies of the lowest levels

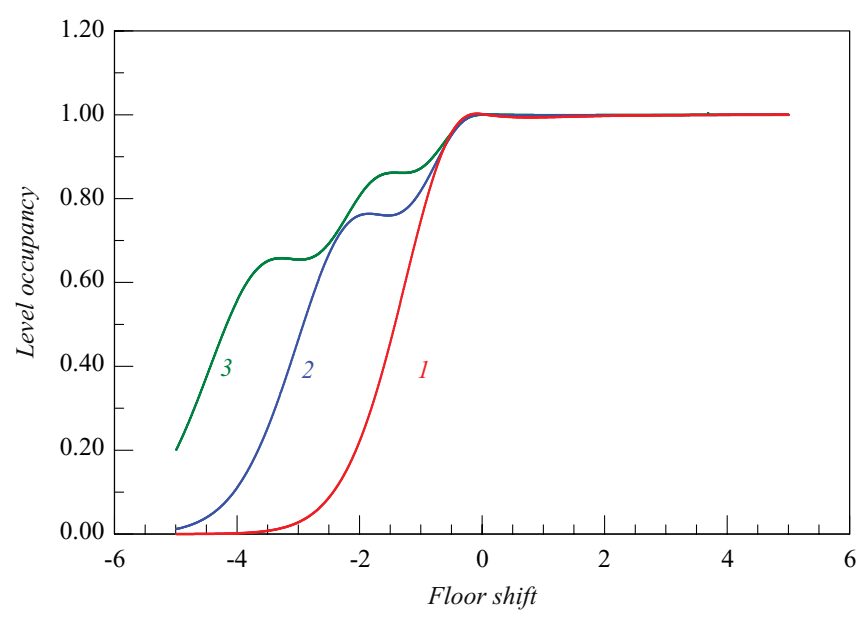

FIG. 4. (Color online) Occupancy of the three lowest gravitational levels as a function of floor shift (step height); quasiclassical calculation. Negative values of the shift correspond to step down, positive to step up. The initial occupancy of all levels in front of the step is equal to 1 . The curves are labeled by the level number. 
at some distinct well-defined values different from those in the original beam. On the other hand, the presence of "hidden" steps down could result in an unanticipated deficiency of particles in the lower states with inevitable errors in interpretation of the experimental data. According to Fig. 4, the accidental steps up are not dangerous in this regard with a caveat that the geometry is open.

It is very difficult to say whether the real experimental setup had such steps or more complicated obstacles. Therefore, the equal occupancy of the gravitational states in the beam entering the rough waveguide cannot be taken for granted. This may be a possible explanation for why in some experiments [1] the occupancy of the lowest gravitational state seemed to be about one-half of the occupancy of higher states. For this to happen, it would be enough for the beam to encounter a $6-\mu \mathrm{m}$ step down on the way to the waveguide.

\section{SUMMARY AND DISCUSSION}

In summary, we investigated the effects of beam preparation and of the properties of the waveguide roughness on the exit neutron count and on the possibility of resolving low-energy quantum states for neutrons bound by the gravity field. There are two major obstacles to a reliable interpretation of experimental data: a lack of experimental data on the initial occupancy of the gravitational states, before the beam enters the waveguide, and insufficient information about the mirror roughness.

We showed that even if the initial occupancy of the ultralowenergy states is uniform when the beam exits the reactor, this uniformity in the occupancy of the gravitational bound states may not always be the case after the beam encounters even benign obstacles, such as mirrors and collimators, on the way to the experimental cell (rough waveguide). This is unfortunate since the particle distribution in the beam in the peV range cannot be routinely measured before the beam enters the waveguide. One of the possible ways to mitigate this uncertainty is to make the beam go over a sufficiently high step up in front of the waveguide.

Since both the mirror roughness and the beam preparation can to a large degree be controlled in experiment, both factors can be used for optimizing the experimental results, that is, for improving the resolution of quantized states with predefined neutron energies. As was shown in the previous section, a creative use of obstacles (steps) in front of the waveguide can rearrange the occupancy distribution $N_{j}(0)$ for neutrons entering the beam and, as a result, affect the exit neutron counts for gravitational states, Eq. (25). The most interesting option here is the possibility of having quantum plateaus for neutrons in various gravitational states as a function of the step height.

The effect of mirror roughness can lead to even more intriguing consequences. We demonstrated that, as far as the exit neutron count is concerned, within certain constraints all the information on the properties of the waveguide, including its length, roughness, absorption threshold, etc., collapses into a single dimensionless constant $\Phi$, Eq. (34). In general, the larger the value of $\Phi$, the more pronounced are the quantum steps on the dependence of the neutron count on the waveguide width $h$. These quantum steps become very clear at $\Phi>40$.
The fact that, as far as the exit neutron count is concerned, all the mirror parameters collapse into a single constant $\Phi$, means that using rough mirrors with different correlation functions of surface inhomogeneities and various values of the correlation radius $R$ is equivalent effectively to just renormalizing the amplitude of roughness $\ell$. This is similar to the result of Ref. [13] that all the normalized curves for the roughnessrestricted conductivity of ultrathin quantized metal films $\sigma(L)$ for different types of surface roughness collapse into a single curve ( $L$ is the film thickness). As in the neutron problem above, here too all the curves $\sigma(L)$ can be made identical just by renormalizing the roughness amplitude, which enters the conductivity as a coefficient.

Of course, as in Ref. [13], the condition of such self-affinity of all curves is that the value of $R$ is not very large and the roughness-driven interstate transitions are robust. At large values of $R$, the roughness-driven transitions between the quantized states become severely depressed and one should observe a set of distinct scales that correspond to the opening of new individual scattering (transition) channels. Traces of this effect can be seen in Ref. [10]. As in quantized metal films, here too the threshold values of the waveguide spacings, which correspond to the opening of new channels, depend on the correlation function of mirror roughness. However, in contrast to metal films for which the roughness is an accident of growth or deposition, for the neutron problem in question, the mirror roughness is man-made and there is no reason to have large values of $R$ : on the contrary, one wants to make the interstate transitions as robust as possible, which requires manufacturing roughness with relatively small values of $\dot{R}$.

In this sense, the use of the rough waveguide to produce neutrons with well-defined quantized energies in the peV range in experiments of this type differs dramatically from most other experiments with transport along rough surfaces. Here one deals with a unique situation when the surface roughness is not a random artifact of wall preparation or growth, but is created artificially and can be easily manipulated to achieve the optimal experimental results. This is not so far-fetched since the spatial scale of desirable roughness is in the $\mu \mathrm{m}$ range.

There are several ways of increasing the value of $\Phi$ by changing the roughness parameters. The simplest and the most straightforward way is, of course, to increase the amplitude of roughness $\eta$. However, this would also degrade both the accuracy of calculations, which assumes that $\eta$ is small, and, more importantly, the accuracy of measurements: any substantial increase in the amplitude of roughness increases the uncertainty in the distance between the mirrors $h$, which is already high, in the range of $10 \%-20 \%$, and the related uncertainty in the positions of the gravitational energy levels [10]. Since one of the main goals of the experiment is to obtain neutrons in the well-defined energy states, the increase in the roughness amplitude $\eta$ has a very limited appeal and should be used only after all other methods of increasing the value of $\Phi$ have been exhausted.

Another way that roughness affects $\Phi$ is via the correlation radius of surface inhomogeneities $r$, Eqs. (36)-(40) and Fig. 2. This is also a relatively straightforward approach since $\Phi$ is inversely proportional to $\sqrt{r}$. However, the decrease in $r$ beyond a certain value is difficult to achieve experimentally. More importantly, the correlation radius $r$ should remain above 
the amplitude of roughness, $r>\eta$, and $r$ and $\eta$ always enter $\Phi$ in the combination $\eta^{2} / \sqrt{r}$, Eqs. (36)-(40).

The most unusual option is to exploit the dependence of $\Phi$ on the shape of the correlation function [or, more precisely, on its Fourier image $\psi(y)$ ], Fig. 3. Note that one needs the information on the shape of the correlation function anyway. The correlation function $\psi(y)$ not only affects $\Phi$ directly, via the integral (34), but it also can distort the experimental measurements of the amplitude and the correlation radius of surface inhomogeneities $\eta$ and $r$. The result of the STM study of a rough metal surface in Ref. [16] showed that the values of $\eta$ and $r$ were vastly different depending on whether the same STM data were fitted using the Gaussian or simple exponential correlation function. This result is even more surprising taking into account the fact that both of these correlation functions, in contrast to the power-law correlators, have well-defined values of the correlation radius and the roughness amplitude.

The inevitable conclusion is that in neutron experiments of the type described above, in which the mirror roughness is created artificially, the best strategy is not to do some random scratching of the mirror and measure the roughness parameters afterward, but to choose the optimal shape of the surface correlator and the roughness parameters beforehand, generate the corresponding roughness profile numerically, and then reproduce this roughness on the mirror surface. Essentially, the suggestion is to start from a computer simulation of the desired roughness profile and then to recreate the corresponding rough grid on the mirror.

Though this looks like a straightforward operation, there is a difficulty associated with the fact that the computational, theoretical, and experimental approaches to surface roughness are significantly different. Theoretically, the surface roughness is more likely than not described via the roughness correlation function $\zeta(x)$ in the same way as has been done above. Computationally, the random roughness $y(x)$ is generated using some choice of the probability distribution function $P[y(x)]$ with little or no regard to the long-range correlations. The experimental approach to roughness depends on the type of experiment: the wave scattering from the rough surface is best described using the correlation function, while the STM and similar measurements are more easily interpreted using the probability distribution. Therefore, to computationally generate and then experimentally reproduce the roughness with desirable correlation properties, it is necessary to outline the corresponding procedure.

In numerical approaches, the rough surface $y(x)$ is generated using some distribution function $P(y)$. The usual choice is the Gaussian distribution,

$$
P(y)=\frac{1}{\sqrt{2 \pi}} \exp \left(-y^{2} / 2\right)
$$

(see Ref. [14] and references therein). Note that the simple distribution $P(y)$ of the type (41) leads to an uncorrelated roughness, $\zeta(x) \propto \delta\left(x-x^{\prime}\right)$. To reproduce meaningful binary correlations $\zeta(x)$,

$$
\zeta(x)=\left\langle y\left(x^{\prime}\right) y\left(x^{\prime}+x\right)\right\rangle_{x^{\prime}} \equiv \frac{1}{L} \int y\left(x^{\prime}\right) y\left(x^{\prime}+x\right) d x^{\prime},
$$

one requires a more complicated distribution $P[y(x)]$.
It is easier to map the link between the theoretical and computational approaches by first discretizing the surface into $N$ segments, $y(x) \rightarrow y_{i}, i=1,2, \ldots, N$, and smoothing the resulting profile after the computations are done. One can start, for example, by generating the surface with a generalized Gaussian probability distribution,

$$
P[\vec{y}]=C \exp \left(-\frac{1}{2} \vec{y} \cdot \widehat{G} \vec{y}\right), \quad \vec{y}=\left(y_{1}, y_{2}, \ldots, y_{N}\right),
$$

with some matrix $\widehat{G}$ that should, in the end, reproduce the desired binary correlation function

$$
\zeta(x) \rightarrow \zeta_{i k}=\zeta(i-k)=\left\langle y_{i} y_{k}\right\rangle=\int y_{i} y_{k} P[\vec{y}] d \vec{y} .
$$

Here $C$ is the normalization constant defined by the equation

$$
1=C \int \exp \left(-\frac{1}{2} \vec{y} \cdot \widehat{G} \vec{y}\right) d \vec{y}
$$

(see below).

If one rotates the vector $\vec{y}$,

$$
\vec{y}=\widehat{A} \vec{g}, \quad \vec{g}=\widehat{A}^{-1} \vec{y},
$$

in such a way as to diagonalize the quadratic form $\vec{y} \cdot \widehat{G} \vec{y}$,

$$
\begin{gathered}
-\frac{1}{2} \vec{y} \cdot \widehat{G} \vec{y}=-\frac{1}{2} \widehat{A} \vec{g} \cdot \widehat{G} \widehat{A} \vec{g}=-\frac{1}{2} \vec{g} \cdot \widehat{A}^{T} \widehat{G} \widehat{A} \vec{g}, \\
\widehat{A}^{T} \widehat{G} \widehat{A}=\widehat{I} \equiv \delta_{i k},
\end{gathered}
$$

the probability distribution (including the Jacobian) becomes

$$
P[\vec{y}] d \vec{y} \rightarrow P[\vec{g}] d \vec{g}=\frac{1}{(2 \pi)^{N / 2}} \exp \left(-\frac{1}{2} \sum_{i=1}^{N} g_{i}^{2}\right)
$$

and all $g_{i}$ become statistically independent,

$$
\left\langle g_{i} g_{k}\right\rangle=\int g_{i} g_{k} P[\vec{g}] d \vec{g}=\delta_{i k} .
$$

The coefficient in Eq. (49), together with the transformation Jacobian, give the normalization coefficient $C$ in Eqs. (43) and (45). Then the roughness correlation function $\widehat{\zeta}=\left\langle y_{i} y_{k}\right\rangle$ acquires the form

$$
\begin{aligned}
\widehat{\zeta} & =\int y_{i} y_{k} P[\vec{y}] d \vec{y}=\int A_{i l} g_{l} A_{k m} g_{m} P[\vec{g}] d \vec{g} \\
& =A_{i l} A_{k m} \delta_{l m}=\left(G^{-1}\right)_{i k}
\end{aligned}
$$

[the last equation is based on Eq. (48)]. Therefore, numerically generating the surface with the given correlation function $\zeta(i-k)$ reduces to inverting the matrix $\widehat{\zeta}$, which corresponds to a given correlation function $\zeta(x)$, and then generating the random surface with the Gaussian distribution (43) with $\widehat{G}=$ $\widehat{\zeta}^{-1}$. Computationally, this is a relatively straightforward task that reduces to inversion of large matrices. After the surface with desired roughness has been generated computationally, this profile can be reproduced on the surface of the mirror. 
Thus, the use of rough waveguides opens the door to many more experimental options than was previously realized.

We are planning to discuss this issue, together with more general issues that arise from the differences between theoretical, experimental, and computational approaches to rough surfaces, in more detail in a separate paper.

\section{ACKNOWLEDGMENTS}

We are grateful to P. Nightingale for helpful discussions concerning generation of random surfaces. One of the authors (A.M.) is grateful to the members of GRANIT group at ILL, and especially to V. Nesvizhevsky, for the hospitality during his visits to Grenoble and for the stimulating discussions.
[1] V. V. Nesvizhevsky et al., Nature (London) 415, 297 (2002); Phys. Rev. D 67, 102002 (2003); Eur. Phys. J. C 40, 479 (2005); for additional references, see also [http://lpscwww.in2p3.fr/ UCN/NiveauxQ_G/publications/index.html].

[2] V. V. Nesvizhevsky, Phys. Usp. 53, 645 (2010).

[3] G. Breit, Phys. Rev. 32, 273 (1928).

[4] J. H. Freed, Ann. Phys. Fr. 10, 901 (1985).

[5] H. Murayama et al. (Particle Data Group), Review of Particle Properties, p. 374 [Phys. Rev. D 66, 010001 (2002)].

[6] V. V. Nesvizhevsky and K. V. Protasov, Class. Quantum Grav. 21, 4557 (2004).

[7] Possible use of gravitational quantization of neutrons for measuring the fundamental forces was the main topic of the workshop [http://lpsc.in2p3.fr/congres/granit06/index.php] (ILL, Grenoble, 2006).

[8] V. V. Nesvizhevsky et al., Nucl. Instrum. Methods Phys. Res. Sect. A 578, 435 (2007).

[9] A. E. Meyerovich and V. V. Nesvizhevsky, Phys. Rev. A 73, 063616 (2006).
[10] R. Adhikari, Y. Cheng, A. E. Meyerovich, and V. V. Nesvizhevsky, Phys. Rev. A 75, 063613 (2007).

[11] A. E. Meyerovich and A. Stepaniants, Phys. Rev. B 60, 9129 (1999).

[12] A. Yu. Voronin, H. Abele, S. Baessler, V. V. Nesvizhevsky, A. K. Petukhov, K. V. Protasov, and A. Westphal, Phys. Rev. D 73, 044029 (2006).

[13] A. E. Meyerovich and I. V. Ponomarev, Phys. Rev. B 65, 155413 (2002); Y. Cheng, and A. E. Meyerovich, ibid. 73, 085404 (2006).

[14] J. A. Ogilvy, Theory of Wave Scattering from Random Surfaces (Adam Hilger, Bristol, 1991).

[15] K. H. Guenther, P. G. Wierer, and J. M. Bennett, Appl. Opt. 23, 3820 (1984); R. M. Feenstra, D. A. Collins, D. Z.-Y. Ting, M. W. Wang, and T. C. McGill, Phys. Rev. Lett. 72, 2749 (1994); Y. P. Zhao, H.-N. Yang, G.-C. Wang, and T.-M. Lu, Appl. Phys. Lett. 68, 3063 (1996).

[16] R. C. Munoz, G. Vidal, G. Kremer, L. Moraga, C. Arenas, and A. Concha, J. Phys.: Condens. Matter 12, 2903 (2000).

[17] V. V. Nesvizhevskii, Phys. Usp. 47(5), 511 (2004). 